\title{
SCIENTIFIC EXHIBIT PRIZES - ANNUAL MEETING 1982
}

\section{LAURÉATS DU CONCOURS DES MONTAGES SCIENTIFIQUES CONGRÉS ANNUEL 1982}

Prizes awarded for the three Scientific Exhibits judged to be the best presented at the Annual Meeting of The Canadian Anaesthetists' Society at Quebec, May 22-26, 1982, were:

First Prize: Dr. Kwok Lee, Department of Anaesthesia, Hospital For Sick Children, and University of Toronto, Toronto, Ontario; "Cardiopulmonary Resuscitation in Children - Modern Concepts."

Second Prize: Dr. R.A. Browne, Department of Anaesthesia, Hamilton General Hospital and McMaster University, Hamilton, Ontario: "Hepatitis B and You."

Third Prize: Dr. B.Y. Ong, Department of Anaesthesia, Health Sciences Centre, University of Manitoba: "An Obstetric Anaesthesia Audit System."
Les prix attribués aux trois meilleurs montages scientifiques preséntés au congrés annuel de la Société Canadienne des Anesthésistes à Québec du 22-26 Mai, 1982, étaient:

Premier Prix: docteur Kwok Lee, Département d'Anesthésie, Hospital for Sick Children, et l'Université de Toronto, Toronto, Ontario: "Réanimation cardiopulmonaire chez les enfants les concepts modernes."

Deuxième Prix: docteur B.A. Browne, Département d'Anesthésie, Hôpital General d'Hamilton et l'université McMaster, Hamilton, Ontario: "L'hepatite B et Vous."

Troisième Prix: docteur B.Y. Ong, Département d'Anésthésie, Centre des sciences de la santé et l'Université de Manitoba, Winnipeg, Manitoba: "Un système pour évaluation de l'anesthésie obstetricale par critères objectifs."

\section{AUdiovisuAL PROGRAMME PRIZE - ANNUAL MEETING 1982}

\section{LAURÉAT DU CONCOURS AUDIOVISUEL - CONGRÉS ANNUEL 1982}

The prize for the best presentation in the audiovisual programme at the Annual Meeting of The Canadian Anaesthetists' Society at Quebec, 22-26 May, 1982 was awarded to Dr. Hilary Taylor, Department of Anaesthesia, York-Finch Hospital, Toronto, for her exhibit "Continuous Infusion Epidural Analgesia In Obstetrics."
Lauréat du concours audiovisuel au congrés annuel de la Sociéte Canadienne des Anesthésistes à Québec du 22 au 26 mai 1982 était docteur Hilary Taylor, Hôpital YorkFinch, Toronto, Ontario, pour sa demonstration "Analgésie péridurale par perfusion continue en l'obstetrique." 